\title{
CONTROLADOR FUZZY MULTIVARIÁVEL APLICADO A UM SIMULADOR DE CIRCUITO DE MOAGEM*
}

\author{
Modesto Pereira Júnior ${ }^{1}$ \\ Thiago Antônio Melo Euzébio²
}

\section{Resumo}

A operação da moagem é de grande importância na mineração, uma vez que é a área da fragmentação que requer maiores investimentos, há maior gasto de energia e seu desempenho afeta diretamente a taxa de recuperação do mineral de interesse. O controle do circuito de moagem é desafiador por se tratar de um sistema multivariável, não-linear e por se caracterizar pela existência de forte acoplamento entre variáveis e diferentes atrasos de tempo. $\mathrm{Na}$ indústria, grande parte desses circuitos é controlada apenas por PIDs. Nesses casos, quando há perturbações no sistema, tais como variação do tamanho das partículas na alimentação ou variação na dureza do minério, a interferência de operadores alterando setpoints é exigida para manter a estabilidade do sistema. Neste trabalho é desenvolvido um controlador fuzzy multivariável aplicado a um simulador de moagem. A camada de controle proposta encontra-se acima da camada regulatória. O objetivo é o de variar dinamicamente os setpoints dos PIDs para maximizar a taxa de produção dentro de uma faixa granulométrica.

Palavras-chave: Controlador fuzzy; Moinho de bolas; Controle de processos; Controle multivariável.

\section{FUZZY MULTIVARIATE CONTROLLER APPLIED TO A GRINDING CIRCUIT SIMULATOR}

\begin{abstract}
The operation of grinding is of great importance in mining, since it is the area of fragmentation that requires larger investments, there is greater energy expenditure and its performance directly affects the rate of recovery of the mineral of interest. The control of the grinding circuit is challenging because it is a multivariate, nonlinear system and because it is characterized by the existence of strong coupling between variables and different time delays. In industry, most of these circuits are controlled only by PIDs. In such cases, when there are disturbances in the system, such as particle size variation in feed or variation in ore hardness, interference from operators changing setpoints is required to maintain system stability. In this paper is developmented an multivariate fuzzy controller applied to an grinding simulator. The control level proposed is found above of the regulatory level. The objective is the vary dynamically the set points of the PIDs to maximize the production rate inside of an particle size range.
\end{abstract}

Keywords: Fuzzy controller; Ball mil; Process control; Multivariate control.

1 Engenheiro Eletricista, Mestrando em Instrumentação, Controle e Automação de Processos de Mineração, Yara Fertilizantes / Instituto Tecnológico Vale / Universidade Federal de Ouro Preto, Ouro Preto, Minas Gerais, Brasil.

2 Engenheiro Eletricista, D. Sc., Pesquisador, Instituto Tecnológico Vale, Ouro Preto, Minas Gerais, Brasil. 


\section{INTRODUÇÃO}

A moagem é a última etapa no processo de cominuição onde as particulas são reduzidas em tamanho por uma combinação de impacto e abrasão. Representa uma importante operação unitária no processamento de minerais em plantas de concentração. $\mathrm{O}$ tamanho das partículas do produto tem grande influência na taxa de recuperação dos minerais de interesse. Uma moagem muito grosseira limita a recuperação, já uma moagem demasiadamente fina pode ser inviável economicamente. No processamento de minérios de cobre, desde a mina até o embarque do concentrado, essa operação unitária contribui com $40 \%$ dos custos totais [1].

O circuito do moinho de bolas é essencialmente um sistema de múltiplas entradas e múltiplas saídas (MIMO), caracterizado pelo acoplamento entre as variáveis do processo, não-linearidades e atrasos de tempo. O controle estável do circuito de moagem é geralmente interrompido por perturbações, tais como o tamanho das partículas na alimentação e mudança na dureza do minério.

O uso de um simulador dinâmico de moagem permite diversas vantagens ao comparativo de estratégias de controle. Pode-se, por exemplo, introduzir perturbações severas ao sistema e variações repentinas do tipo de minério processado no moinho. É possível também modificar características físicas dos equipamentos e o número de variáveis de processo e manipuladas. Todas essas alterações são feitas em tempo de simulação, a custo zero e sem risco de danos aos equipamentos.

Alguns trabalhos de pesquisa foram publicados sobre estratégias de controle para circuitos de moagem. Esses métodos de controle envolvem controle adaptativo baseado em matriz dinâmica [2], controle preditivo por modelo (MPC) [3, 4] e controle fuzzy $[5,6]$. Estes trabalhos apresentaram desempenho superior ao dos controladores PID e produziram menores oscilações após as alterações de setpoint. Também apresentaram menor variabilidade no produto final da moagem. No entanto, nos trabalhos revisados, não houve a análise do comportamento dos controladores durante uma variação na granulometria do material, situação que ocorre frequentemente nas plantas de beneficiamento.

Neste artigo é apresentado o desenvolvimento e a aplicação de um controlador fuzzy multivariável para um circuito de moagem simulado, atuando em uma camada de controle acima da camada regulatória. O desempenho do controlador proposto é comparado aos controladores PIDs, cuja aplicação é a mais comum na indústria. Os objetivos do controlador proposto são maximizar a taxa de alimentação de minério novo e manter a distribuição do tamanho das partículas dentro de uma faixa de operação.

\section{CIRCUITO DE MOAGEM SIMULADO}

O simulador utilizado para o desenvolvimento deste trabalho possui uma estrutura de modelo aberto e abordagem modular. A partir de uma biblioteca de equipamentos, modelos de interconexão e sensores, pode-se agrupar estes itens de modo a construir o modelo da planta a ser simulada. As equações matemáticas que descrevem cada componente podem ser verificadas em [7]. Trata-se de um simulador dinâmico, em que se pode investigar o efeito de alterações na moabilidade do minério e outros parâmetros para o sistema de moagem e 
classificação existente. A grande vantagem de se utilizar um simulador dinâmico é que podemos obter a resposta transitória do circuito e alterar todos os parâmetros, desde parâmetros construtivos dos equipamentos até parâmetros do minério.

$\mathrm{O}$ circuito de moagem simulado consiste em um moinho de bolas, um tanque, e um hidrociclone como ilustrado na Figura 1. A moagem é realizada no interior do moinho pela ação de bolas de aço sobre o minério. E adicionada água à alimentação de minério, para se criar uma polpa, de forma a promover a moagem e um fluxo através do moinho. O tanque serve para ajustar a densidade da polpa e promover sua homogeneização. Uma bomba de polpa com velocidade variável é utilizada para transferir o material até o hidrociclone, que realiza a classificação do produto. $O$ material que está em uma faixa de tamanho adequado segue para a próxima etapa do processo (produto) e o material de tamanho inadequado retorna ao moinho para ser moído novamente. Os principais parâmetros técnicos do moinho de bolas simulado são apresentados na Tabela 1.

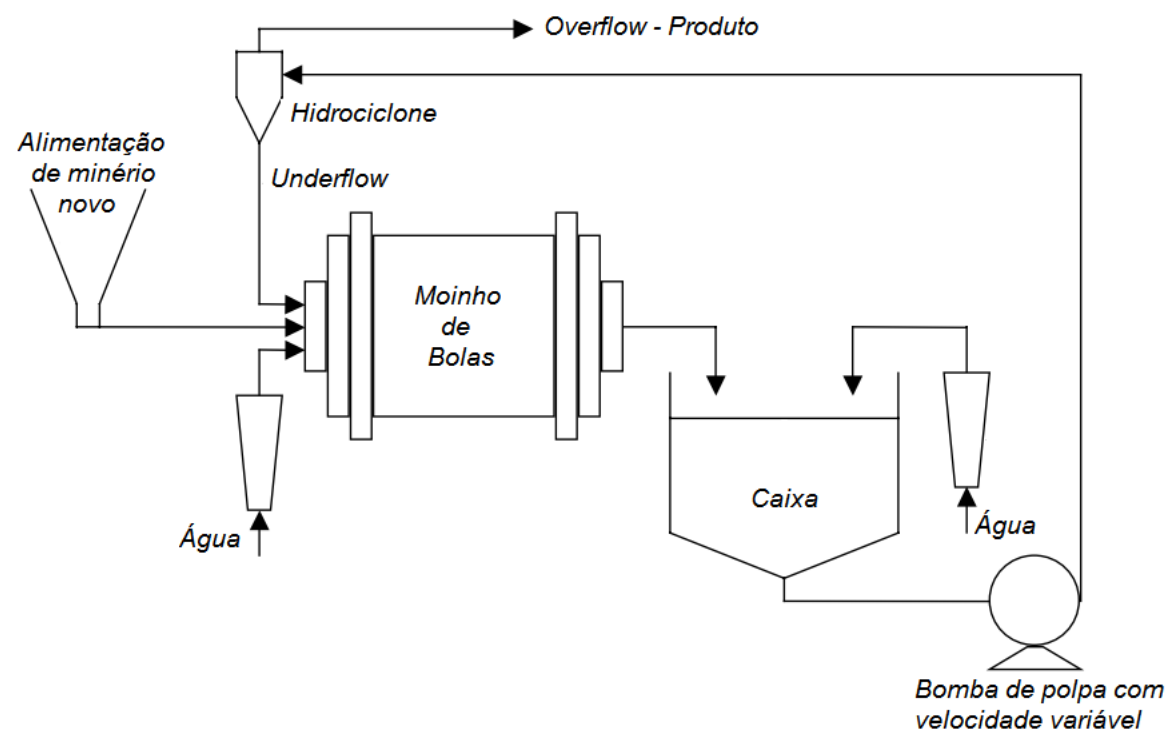

Figura 1. Circuito de moagem estudado.

Tabela 1. Parâmetros técnicos do moinho de bolas simulado

\begin{tabular}{|l|l|}
\hline Comprimento [m] & 2,44 \\
\hline Diâmetro[m] & 2,44 \\
\hline Tipo de descarga & Overflow \\
\hline $\begin{array}{l}\text { Tamanho máximo de sólidos (alimentação) } \\
\text { [mm] }\end{array}$ & 1,0 \\
\hline Rotação [RPM] & 21,3 \\
\hline \% da velocidade crítica & 76,1 \\
\hline Granulometria do Produto [\%, - 200 mesh] & $72 \pm 2$ \\
\hline
\end{tabular}

No simulador utilizado, o modelo para moinho de bolas baseia-se no balanço de massa para o material em cada intervalo de tamanho, seguindo o princípio da mistura perfeita. Essas equações podem ser escritas em forma de vetor como:

$$
\frac{d m(t)}{d t}=\mathrm{f}(t)-\mathrm{p}(t)-\gamma(t)(\phi[\mathrm{S}-\mathrm{BS}] \mathrm{m}(t))
$$


onde $\mathrm{m}(\mathrm{t})$ é o vetor de fração de massa no interior do moinho, $\mathrm{f}(\mathrm{t})$ é o vetor de fração de massa na carga do moinho, $\mathrm{p}(\mathrm{t})$ é o vetor de fração de massa do produto da moagem, $\gamma$ é a dureza do minério, $\varphi$ representa a fração de primeira ordem da cinética assumida, B e S são as matrizes de quebra.

\section{ESTRATÉGIAS DE CONTROLE}

\subsection{Controlador PID}

Controladores PID possuem larga aplicação em processos industriais. Isto se deve, em grande parte, ao seu bom desempenho observado quando aplicado em malha fechada a processos com dinâmica conhecida. Sua facilidade de implantação e manutenção favorece a sua implementação em malhas de controle com uma única variável a ser controlada.

A lógica de controle baseia-se no cálculo do erro e $(\mathrm{t})$ entre o valor desejado $r(\mathrm{t})$ e o retorno da planta $\mathrm{y}(\mathrm{t})$, isto é, $\mathrm{e}(\mathrm{t})=\mathrm{r}(\mathrm{t})-\mathrm{y}(\mathrm{t})$. Deste modo, a lei de controle do controlador ilustrado na Figura 2, em tempo contínuo, é descrita pela equação:

$$
C=k_{p} e(t)+k_{i} \int_{0}^{t} e(t) d t+k_{d} \frac{d e(t)}{d t}
$$

onde, Kp, Ki, Kd são os ganhos proporcional, integral e derivativo do PID. A seleção ótima dos ganhos é realizada utilizando métodos de sintonia existentes na literatura, como Método de Ziegler e Nichols, Método de Cohen Coon, Método do Modelo Interno (IMC), entre outros. Uma compilação de métodos de sintonia PID pode ser visto em [8].

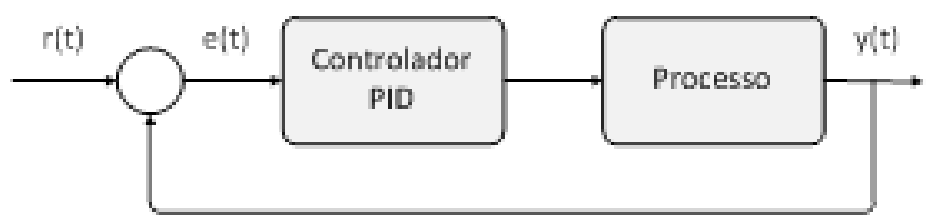

Figura 2. Controle PID aplicado em malha fechada.

Para aplicar o controle PID no simulador de moagem, utilizaram-se os modelos da planta apresentados na seção anterior. A partir desses modelos aplicou-se o método de sintonia IMC para identificar os valores dos parâmetros de sintonia $\mathrm{Kp}, \mathrm{Ki}$ e $\mathrm{Kd}$. As malhas criadas foram: controle da taxa de alimentação de minério, controle da taxa de adição de água no moinho e controle de densidade da polpa. Os valores dos parâmetros utilizados são apresentados na Tabela 2.

Tabela 2. Parâmetros dos controladores PID

\begin{tabular}{|c|c|}
\hline Malha & Parâmetros \\
\hline \multirow{2}{*}{ Taxa de alimentação de minério } & $\mathrm{K}_{\mathrm{p}}: 0,0$ \\
\hline & $K_{i}: 3,0$ \\
\hline \multirow{2}{*}{$\begin{array}{l}\text { Taxa de adição de água no } \\
\text { moinho }\end{array}$} & $\mathrm{K}_{\mathrm{p}}: 1,0$ \\
\hline & $\mathrm{K}_{\mathrm{i}:}: 10,0$ \\
\hline \multirow{2}{*}{ Densidade da polpa } & $K_{p}: 0,1$ \\
\hline & $\mathrm{K}_{\mathrm{i}}: 6,0$ \\
\hline
\end{tabular}




\subsection{Controlador Fuzzy}

Os controladores industriais baseados em lógica fuzzy podem ser projetados a partir do conhecimento experimental de operadores humanos. A partir de um conjunto de regras linguísticas que descrevem as estratégias de controle, um algoritmo é construído onde as palavras são definidas como conjunto fuzzy. As principais vantagens desta aproximação são a possibilidade de implementar regras baseadas na experiência, na intuição, na heurística e no fato de que não é necessário um modelo do processo [9].

Na Figura 3 é ilustrado um controlador fuzzy típico. A etapa de fuzzyficação é o mapeamento do domínio de números reais (variáveis do processo) para o domínio fuzzy. Cada variável é convertida em uma variável linguística. Por exemplo, uma taxa de alimentação de minério de 1 t/h torna-se taxa de alimentação de minério baixa.

Obtidas as entradas fuzzy, o sistema de decisão deverá realizar as inferências necessárias para gerar as saídas dos conjuntos fuzzy, baseado no conhecimento armazenado. Essa etapa de inferência consiste na avaliação das variáveis antecedentes pelas regras estabelecidas. Para as regras de natureza condicional, é utilizada a seguinte sintaxe:

\section{SE "Percentual de sólidos" é Baixo ENTÃO "Taxa de adição de água no moinho" é Baixa. \\ SE "Percentual de sólidos" é Alto ENTÃO "Taxa de adição de água no moinho" é Média.}

Onde a condição é um estado característico do processo ou uma combinação lógica de estados característicos e o resultado é uma operação.

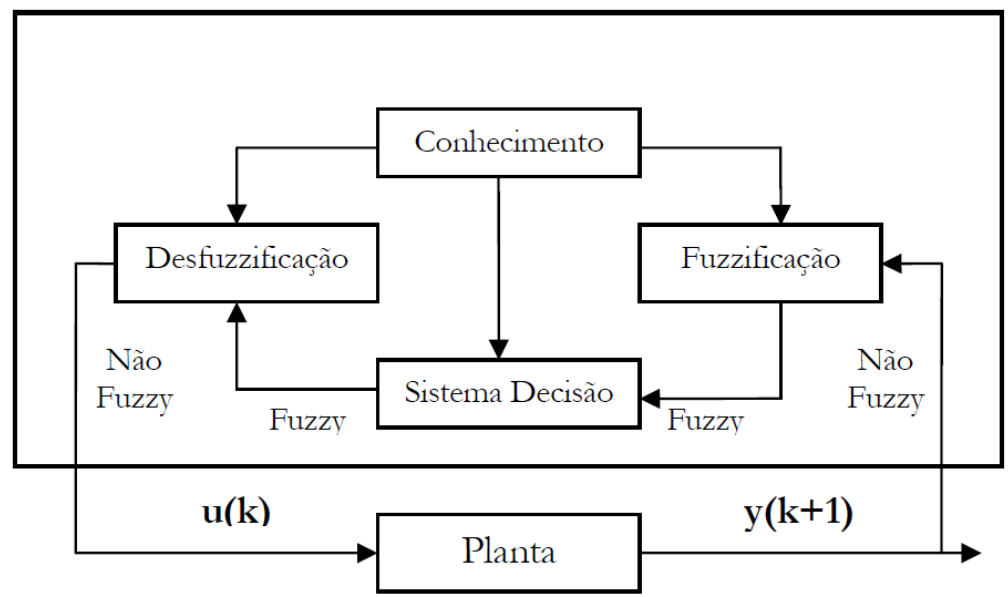

Figura 3. Diagrama típico de um controlador fuzzy.

Já a etapa de defuzzyficação consiste em transformar a saída em domínio fuzzy para o domínio real, já que um elemento de controle (por exemplo, um atuador) não pode processar diretamente esta informação fuzzy.

Foi desenvolvido um controlador fuzzy multivariável que atua em uma camada de controle acima da camada regulatória para alterar o setpoints dos controladores PIDs. Essa estratégia tem por objetivo atingir a qualidade requerida pelo processo e maximizar a taxa de alimentação de minério, mesmo sob perturbações. 
A partir das variáveis de processo granulometria, percentual de sólidos e carga circulante são calculados os setpoints para as variáveis manipuladas taxa de alimentação de minério, taxa de adição de água no moinho e densidade da polpa.

O conhecimento utilizado pelo controlador fuzzy foi baseado no resultado de entrevistas com operadores de sala de controle de uma mineradora no estado de Minas Gerais. Esses operadores possuem grande experiência em plantas de beneficiamento de minério e manipulam os setpoints observando o resultado nas variáveis de controle. Abaixo são listadas as principais regulações a que o sistema obedece:

Regra 1: Durante a partida do circuito as variáveis controladas carga circulante e percentual de sólidos tem valor baixo, a variável granulometria tem valor alto devido ao transitório. As variáveis manipuladas taxa de alimentação de minério, taxa de adição de água no moinho e densidade da polpa tem os setpoints baixo, para que o tempo de acomodação das variáveis controladas seja o menor possível.

Regra 2: Se as variáveis carga circulante e percentual de sólidos têm valores médios, então a taxa de alimentação de minério tem o setpoint alterado para médio. O aumento da taxa de alimentação de minério propicia o aumento da carga circulante, que por sua vez adiciona partículas mais finas ao moinho (underflow do hidrociclone). Os setpoints da taxa de adição de água no moinho e densidade da polpa são mantidos no nível baixo. A alteração do setpoint de apenas uma variável tem o objetivo de promover alterações suaves nos pontos de operação do circuito.

Regra 3: Se as variáveis carga circulante e a granulometria do produto têm valor alto, mas ainda não temos o percentual de sólidos alto, deve-se aumentar a taxa de alimentação. O setpoint de taxa de adição de água no moinho permanece baixo, pois seu aumento levaria a diminuição do percentual de sólidos. Dessa maneira, granulometria do produto, carga circulante e percentual de sólidos serão altos.

Regra 4: Se as três variáveis controladas estiverem atingido valores médios, podemos aumentar o setpoint de taxa de alimentação de minério. O setpoint deve ser aumentado de maneira que não cause sobrecarga no moinho. Por consequência a taxa de adição de água no moinho é aumentada para controlar o percentual de sólidos. O setpoint de densidade da polpa é mantido no mesmo nível, de modo a manter a carga circulante estável.

Regra 5: Caso a granulometria esteja abaixo do setpoint desejado deve-se diminuir o setpoint da taxa de alimentação de minério e adição de água no moinho. 0 setpoint de densidade da polpa também deve ser diminuído para diminuir a quantidade de sólidos que chega ao hidrociclone.

$\mathrm{Na}$ tabela 3 são apresentadas as descrições das variáveis utilizadas pelo controlador. A tabela 4 apresenta o estado das variáveis e a tabela 5 a formulação das principais regras.

Tabela 3. Descrição das variáveis

\begin{tabular}{|c|l|}
\hline Símbolo & \multicolumn{1}{|c|}{ Descrição da variável } \\
\hline $\mathrm{G}_{\mathrm{p}}$ & $\begin{array}{l}\text { Granulometria (erro entre } \mathrm{a} \\
\text { variável controlada e o setpoint) }\end{array}$ \\
\hline$\%_{\mathrm{s}}$ & Percentual de sólidos \\
\hline $\mathrm{C}_{\mathrm{c}}$ & Carga circulante \\
\hline $\mathrm{T}_{\mathrm{m}}$ & Taxa de alimentação de minério \\
\hline $\mathrm{T}_{\mathrm{a}}$ & Taxa de adição de água no moinho \\
\hline $\mathrm{D}_{\mathrm{p}}$ & Densidade da polpa \\
\hline
\end{tabular}

Tabela 4. Estados das variáveis

\begin{tabular}{|l|l|l|l|}
\hline$№$ & \multicolumn{1}{|c|}{ Estado } & № & \multicolumn{1}{|c|}{ Estado } \\
\hline$S^{1}$ & $G_{p}$ é baixa & $S^{11}$ & $T_{m}$ é alta \\
\hline$S^{2}$ & $G_{p}$ é média & $S^{12}$ & $T_{a}$ é muito baixa \\
\hline$S^{3}$ & $G_{p}$ é alta & $S^{13}$ & $T_{a}$ é baixa \\
\hline$S^{4}$ & $\%_{s}$ é baixo & $S^{14}$ & $T_{a}$ é média \\
\hline$S^{5}$ & $\%_{s}$ é alto & $S^{15}$ & $T_{a}$ é alta \\
\hline$S^{6}$ & $C_{c}$ é baixa & $S^{16}$ & $D_{p}$ é muito baixa \\
\hline$S^{7}$ & $C_{c}$ é alta & $S^{17}$ & $D_{p}$ é baixa \\
\hline$S^{8}$ & $T_{m}$ é muito baixa & $S^{18}$ & $D_{p}$ é média \\
\hline$S^{9}$ & $T_{m}$ é baixa & $S^{19}$ & $D_{p}$ é alta \\
\hline$S^{10}$ & $T_{m}$ é média & & \\
\hline
\end{tabular}


Tabela 5. Regras para controlador fuzzy

\begin{tabular}{|c|llllllllll|}
\hline Número & \multicolumn{3}{|c|}{$\begin{array}{c}\text { Condição } \\
\text { (Nível das var. cont.) }\end{array}$} & \multicolumn{3}{c|}{$\begin{array}{c}\text { Ação } \\
\text { (Setpoint das var. man.) }\end{array}$} \\
\hline$R^{1}$ & $S^{3}$ & $\&$ & $S^{4}$ & $\&$ & $S^{6}$ & $S^{9}$ & $\&$ & $S^{13}$ & $\&$ & $S^{17}$ \\
\hline$R^{2}$ & $S^{3}$ & $\&$ & $S^{4}$ & $\&$ & $S^{7}$ & $S^{10}$ & $\&$ & $S^{13}$ & $\&$ & $S^{17}$ \\
\hline$R^{3}$ & $S^{2}$ & $\&$ & $S^{4}$ & $\&$ & $S^{7}$ & $S^{10}$ & $\&$ & $S^{13}$ & $\&$ & $S^{18}$ \\
\hline$R^{4}$ & $S^{2}$ & $\&$ & $S^{5}$ & $\&$ & $S^{7}$ & $S^{11}$ & $\&$ & $S^{14}$ & $\&$ & $S^{18}$ \\
\hline$R^{5}$ & $S^{1}$ & & & & & $S^{9}$ & $\&$ & $S^{12}$ & $\&$ & $S^{16}$ \\
\hline
\end{tabular}

\section{RESULTADOS E DISCUSSÃO}

O objetivo do controlador no circuito de moagem é manter a granulometria do produto no intervalo $72 \pm 2 \%$ (percentual passante na malha de 200 mesh) e ao mesmo tempo buscar a máxima taxa de alimentação de minério. Em dois instantes de tempo distintos foram aplicadas perturbações no minério que alimentava o circuito simulado. As características do minério são apresentados na tabela 6 .

Tabela 6. Características do Minério

\begin{tabular}{|c|c|}
\hline Instante de tempo [s] & $\%-200$ mesh \\
\hline 0 & 12,64 \\
\hline 3000 & 0,50 \\
\hline 4500 & 15,40 \\
\hline
\end{tabular}

Na figura 4 é apresentada a variável mais importante a ser controlada no circuito de moagem, a granulometria do produto. Pode-se observar que tanto o controlador PID, quanto o controlador fuzzy mantém esta variável dentro do intervalo requerido pelo processo [2000 $<t<3000 \mathrm{~s}$ ]. No instante $t=3000 \mathrm{~s}$ é aplicada a perturbação simulando um minério mais grosso na alimentação do circuito. A granulometria do circuito controlado pelo PID se mantém estável em um valor abaixo do limite inferior [4000 $<\mathrm{t}<4500 \mathrm{~s}$ ] enquanto o controlador fuzzy mantém a variável no intervalo requerido. No instante $t=4500 \mathrm{~s}$ é aplicada nova perturbação no circuito, simulando um minério mais fino na alimentação. Novamente o controlador PID não consegue manter a granulometria dentro do intervalo requerido e o controlador fuzzy mantém a granulometria estável dentro do intervalo requerido.

$\mathrm{Na}$ figura 5 é apresentada a variável minério produzido. Observamos nas três condições de operação o desempenho superior do controlador fuzzy. No instante [2000 < $\mathrm{t}<3000$ ] a produção da moagem com o controlador fuzzy aplicado é 5,4\% maior comparada a produção do circuito controlado pelo PID. Nos instantes [4000 < t $<5000]$ e $[7000<\mathrm{t}<8000]$ a produção da moagem com o controlador fuzzy aplicado é $4,7 \%$ e $2,6 \%$ maior respectivamente.

Na figura 6 é apresentada a variável controlada percentual de sólidos na polpa. A quantidade de água usada no moinho para formar a polpa depende da granulometria da alimentação e do tipo de moinho. A utilização de polpa muito diluída resulta numa moagem pouco eficiente, pois as partículas sólidas se encontram muito dispersas na polpa, sendo poucos os choques efetivos entre as partículas e as bolas. Elevandose a percentagem de sólidos, há um aumento na eficiência de moagem com uma 
redução considerável no consumo de bolas. Essa melhora vai até um certo ponto quando então a eficiência começa decrescer. Portanto, a capacidade de moagem passa por um ponto máximo que corresponde a uma determinada concentração de sólidos na polpa do moinho [10]. Nos primeiros instantes verificamos um percentual de sólidos menor no circuito onde o controlador fuzzy foi aplicado, resultado da regulação $n^{\circ}$ 1, que prevê na partida uma manipulação mais conservadora das variáveis. Decorrido algum tempo, o percentual de sólidos na polpa aumenta e se mantém maior no circuito onde foi aplicado o controlador fuzzy se comparado ao circuito controlado pelo PID. Entre os instantes [3000 $<\mathrm{t}<4500$ ] observamos um aumento no percentual de sólidos que é gerado pelo minério mais grosseiro que alimenta o circuito. Analogamente, entre os instantes [4500 $<\mathrm{t}<8000]$ o percentual de sólidos é ligeiramente menor que o primeiro intervalo devido a alimentação com minério mais fino.

A figura 7 ilustra o comportamento da variável controlada carga circulante. A carga circulante é um artifício utilizado para acertar a distribuição granulométrica do produto do moinho. Ao aumentar-se a carga circulante, aumenta-se a quantidade de material que passa pelo moinho e diminui-se o tempo de residência de cada partícula dentro dele. Em consequência, diminui-se a geração de finos [2]. Nas três condições de alimentação do circuito a carga circulante onde o controlador fuzzy está atuando é maior. Após as perturbações a diferença entre o valor da carga circulante entre os circuitos é maior. $\mathrm{O}$ aumento da carga circulante é um dos fatores que garante ao circuito onde o controlador fuzzy foi aplicado manter o granulometria do produto dentro do intervalo requerido pelo processo.

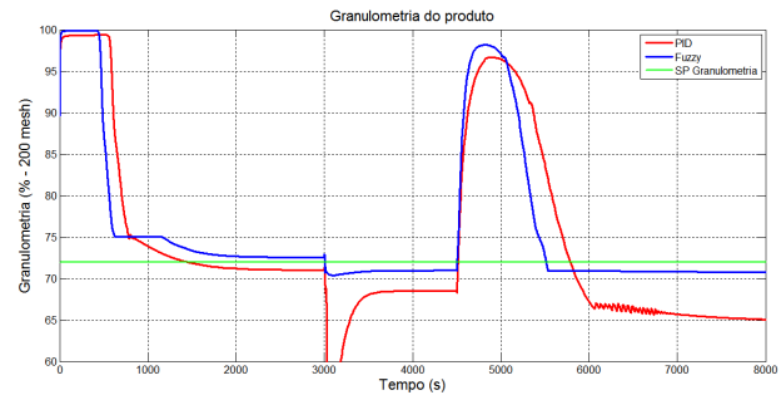

Figura 4. Granulometria do produto.

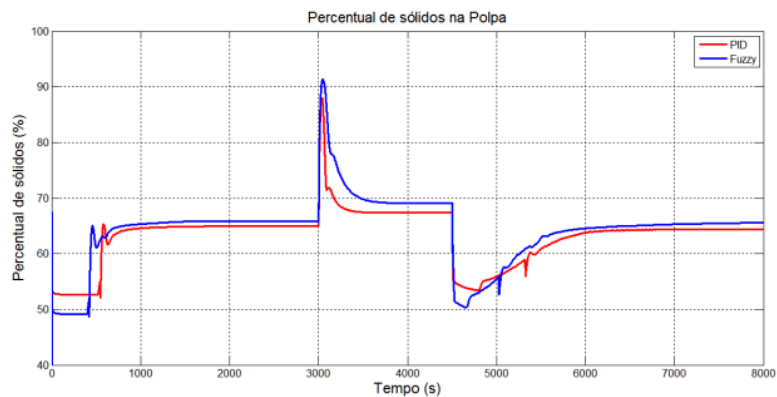

Figura 6. Percentual de sólidos na polpa.

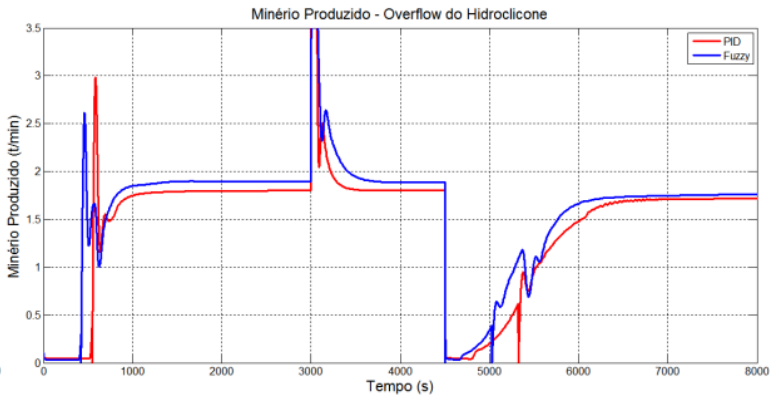

Figura 5. Minério Produzido.

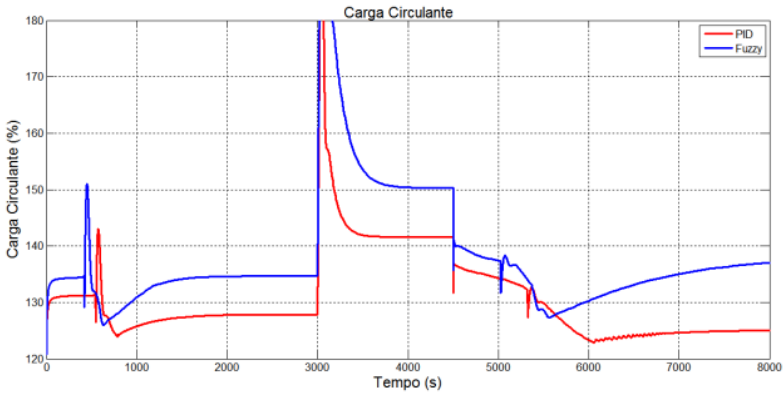

Figura 7. Carga Circulante.

$\mathrm{Na}$ figura 8 pode-se observar o setpoint fixo do controlador PID e o controlador fuzzy variando o setpoint da taxa de alimentação de minério. As maiores variações deste setpoint ocorrem nos momentos em que são aplicados as perturbações $[\mathrm{t}=3000 \mathrm{e} t$ $=4000]$.

$\mathrm{Na}$ figura 9 observa-se o setpoint estabelecido pelo controlador fuzzy maior que o setpoint do controlador PID durante a partida, o que ocasionou um menor percentual 
de sólidos na polpa observado através da figura 6 . Até o instante [ $t=4500$ ] 0 setpoint estabelecido pelo controlador fuzzy é menor que o setpoint do PID, para que se obtenha um percentual de sólidos maior na polpa. Entre [4500 $<t<5500] \mathrm{o}$ setpoint da taxa de adição de água no moinho é maior que o setpoint do controlador PID porque o setpoint da taxa de alimentação de minério foi aumentado. Após o instante [ $\mathrm{t}=5500]$ o setpoint da taxa de adição de água volta a ser menor que o setpoint do PID para que se obtenha um maior percentual de sólidos no polpa e a moagem seja favorecida.

A figura 10 ilustra o comportamento da variável manipulada taxa de adição de água na caixa. Para esta malha de controle temos dois controladores PID em cascata, onde o primeiro atua sobre o setpoint de densidade da polpa e o segundo atua sobre a variável manipulada taxa de adição de água na caixa. Pode-se perceber que no caso do controlador fuzzy é necessário adicionar uma maior quantidade de água à polpa, para que se tenha a mesma densidade, já que tem-se um maior percentual de sólidos.

A figura 11 ilustra o comportamento da variável densidade da polpa. Em todo o período observado a densidade da polpa para o controlador fuzzy é menor e esta diferença máxima é da ordem de $2,8 \%$.

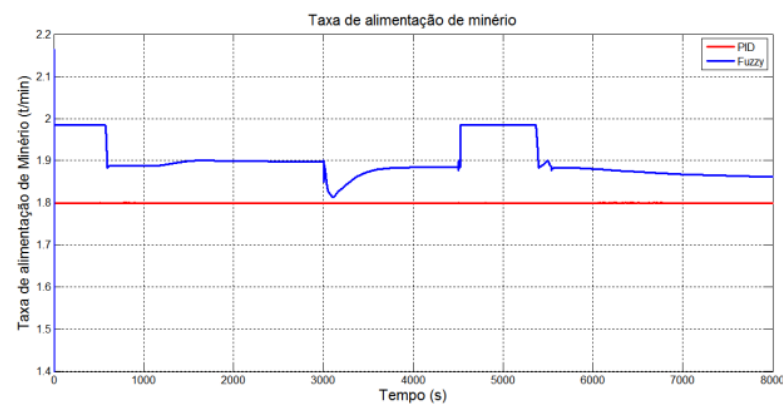

Figura 8. Taxa de alimentação de minério.

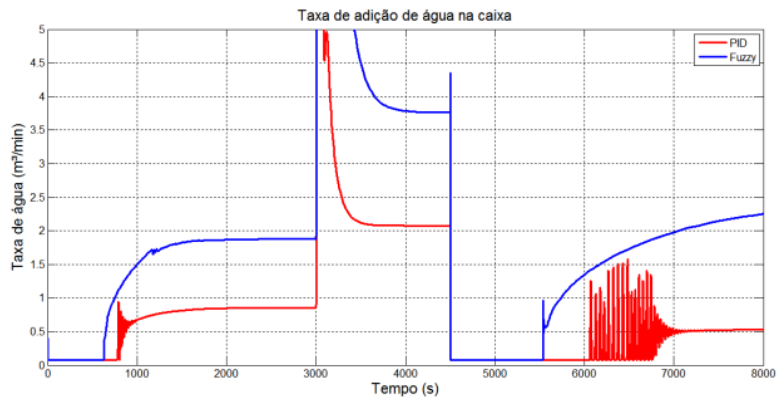

Figura 10. Taxa de adição de água na caixa.

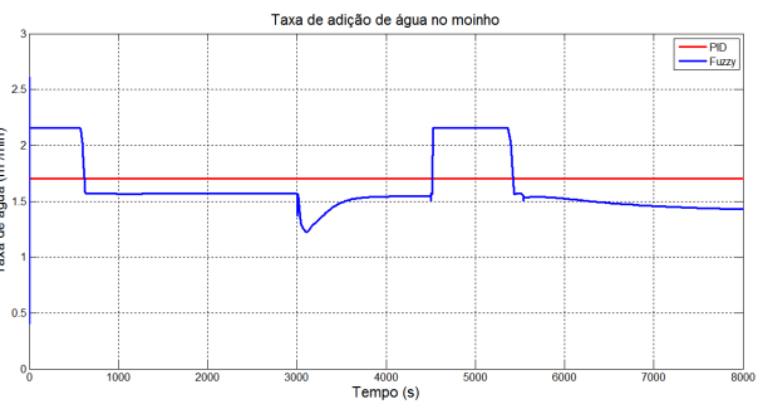

Figura 9. Taxa de adição de água no moinho.

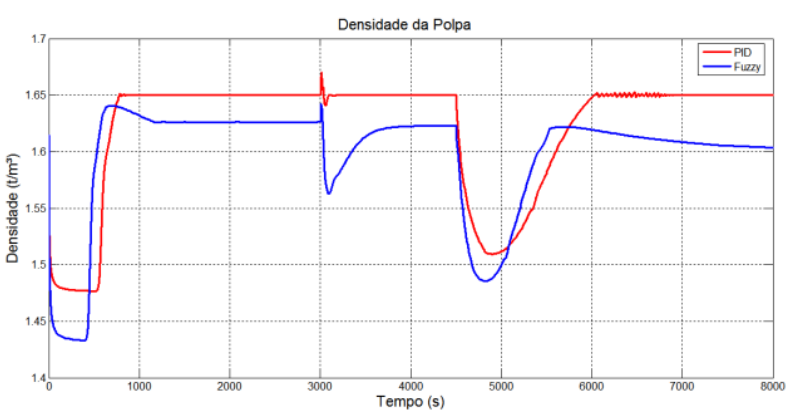

Figura 11. Densidade da polpa.

Na figura 12 são demonstradas as variações nos valores de setpoint para cada uma das variáveis controladas. É importante destacar que apesar das formas de onda apresentarem formas semelhantes, as amplitudes são diferentes. 


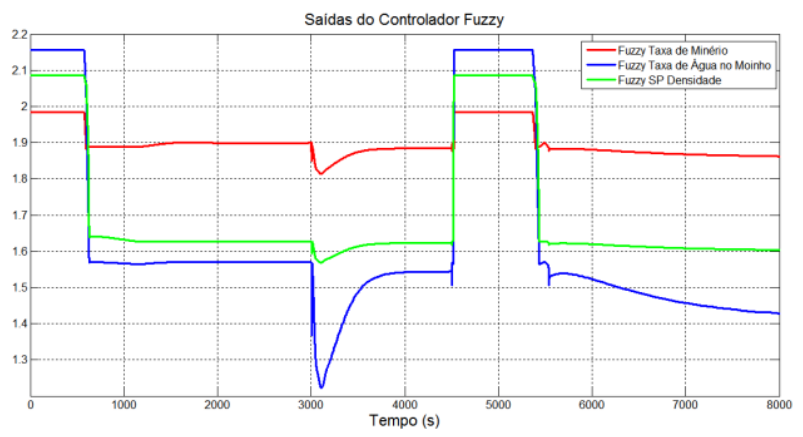

Figura 12. Saídas do controlador fuzzy.

\section{CONCLUSÃO}

O controlador fuzzy multivariável foi desenvolvido para controlar a granulometria do produto no circuito de moagem e ao mesmo tempo buscar a maior taxa de alimentação de minério possível. Atuando em uma camada de controle superior ao controle regulatório, o controlador fuzzy determinou os setpoints para que os objetivos fossem atendidos. A principal variável controlada em um circuito de moagem, a granulometria do produto, foi mantida no intervalo requerido durante 0 período observado. Já o controlador PID não conseguiu manter a variável controlada neste intervalo, quando foram aplicadas perturbações ao sistema, porque o comportamento dinâmico do sistema controlado foi alterado pelas perturbações. Aliado a estabilidade da granulometria do produto, com a aplicação do controlador fuzzy foi possível aumentar a produção de minério na moagem em valores entre $3 \%$ e $5 \%$ para as situações estudadas.

\section{Agradecimentos}

Os autores gostariam de agradecer à equipe do Instituto Tecnológico Vale e da Universidade Federal de Ouro Preto pelo apoio no desenvolvimento deste trabalho. Este trabalho foi parcialmente financiado pela CAPES, Vale S.A. e FAPEMIG.

\section{REFERÊNCIAS}

1 Chaves, Arthur Pinto; Clarck, Antônio Eduardo. Britagem, Peneiramento e Moagem. São Paulo: Oficina de Textos, 2012.

2 Chen, X. song, Li, S. h., Zhai, J. yong, \& Li, Q. Expert system based adaptive dynamic matrix control for ball mill grinding circuit. Expert Systems with Applications. 2009;36(1): 716-723.

3 Ramasamy, M., Narayanan, S. S., \& Rao, C. D. P. Control of ball mill grinding circuit using model predictive control scheme. Journal of Process Control. 2005;15(3):273283.

4 Chen, X. Song, Zhai, J. Yong, Li, S. Hua, \& Li, Q. (2007). Application of model predictive control in ball mill grinding circuit. Minerals Engineering. 2007;20:1099-1108.

5 Chen, Xisong; Zhai, Junyong; Fei, Shumin; Li, Qi. Fuzzy logic based on-line efficiency optimization control of a ball mill grinding circuit. IEEE Fourth International Conference on Fuzzy Systems and Knowledge Discovery. 2007;2:575-580. 
6 Ruel, Michel. Fuzzy Logic Control On a SAG Mill. IFAC Proceedings Volumes. 2013;46: 282-287.

7 Sbárbaro, Daniel; Del Villar, Rene. Advanced Control and Supervision of Mineral Processing Plants. Londres: Springer, 2010.

8 O'Dwyer, Aidan. Handbook of PI and PID controller tuning rules. $3^{\text {rd }}$ Edition. Londres: Imperial College Press, 2009.

9 Balbino, ES. Aumento na eficiência de operação de máquina de mineração usando tecnologia fuzzy. Pontifícia Universidade Católica de Minas Gerais. 2007 [acesso em 30 Mai. 2017]. Disponível em http://www.biblioteca.pucminas.br/teses/EngEletrica_ Balbino ES_1.pdf.

10 Luz, Adão Benvindo da; Sampaio, João Alves; França, Silvia Cristina Alves.

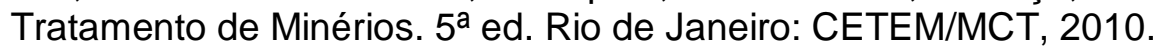

